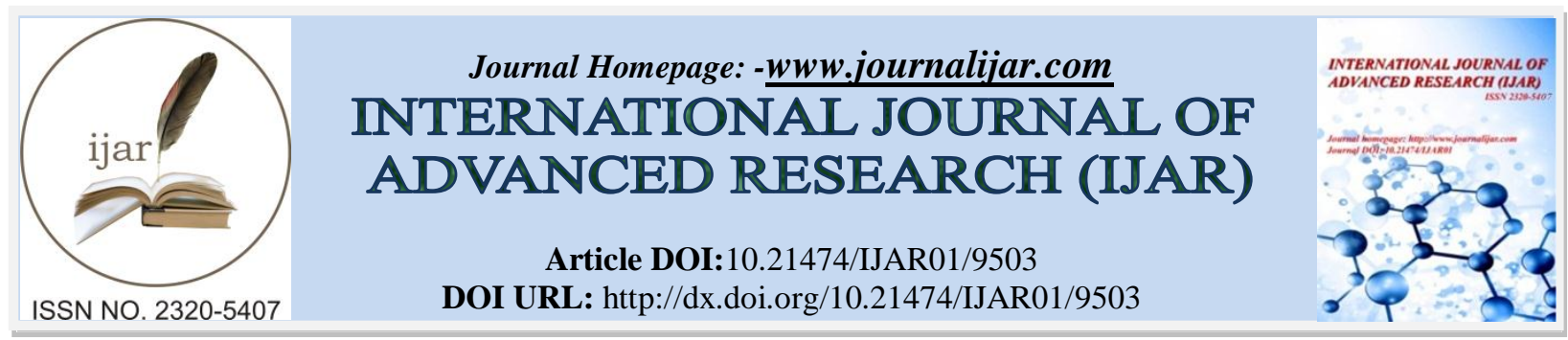

RESEARCH ARTICLE

\title{
CONTINUOUS ULTRASOUND GUIDED ERECTOR SPINAE PLANE BLOCK IN PEDIATRIC LIVER HYDATID CYST SURGERY.
}

\author{
Yasser Asmai, Abdelghafour Elkoundi, Aziza Bentalha, Ismail Bouzekraoui, Ahlam Mosadik, Salma Esherif \\ El Kettani And Alae El Koraichi. \\ Pediatric ICU, Children Hospital, Mohamed V University, Faculty of Medicine and Pharmacy, Rabat, Morocco.
}

\section{Manuscript Info}

Manuscript History

Received: 06 June 2019

Final Accepted: 08 July 2019

Published: August 2019

\begin{abstract}
Background and Objectives: Liver hydatid cyst surgery is considered an extremely painful procedure. Managing pain in this surgery is challenging with several options available, each with limitations. Erector spinae plane block is a novel technique with promising results that have been reported in different types of surgery.

Case Report: We are reporting here the continuous ESP block as a primary mode of postoperative analgesia in a 9-year-old-female scheduled for a liver hydatid cyst surgery. After general anesthesia induction, a continuous erector spinae plane block at T6 level was performed with bupivacaine $0.25 \%$. The intraoperative opioid and anesthetic requirement was very less with a good hemodynamic stability as well as long-lasting postoperative analgesia.

Conclusion: The ESP block is an effective option for liver hydatid cyst surgery. The opioid- and anesthetic-sparing effects exhibited in this case facilitated rapid postoperative recovery.
\end{abstract}

Copy Right, IJAR, 2019,. All rights reserved.

\section{Introduction:-}

Analgesia using an epidural catheterization is still considered the gold standard for postoperative pain management in major open abdominal surgeries ${ }^{1}$. Nonetheless, not all anesthesiologists feel comfortable using this analgesia technique because of its side effects such as hypotension, motor blockade, epidural hematoma and abscess 2,3 . Regional anesthetic techniques would seem a better choice for improving acute pain management in these patients. Erector spinae plane block (ESP) is a novel technique with promising results that have been reported in different types of surgery ${ }^{4,5}$. We describe the case of a successful continuous erector spinae plane block to manage the analgesia during the perioperative procedure, and for pain relief in the postoperative period of a pediatric liver hydatid cyst surgery.

\section{Case Presentation}

The patient was a young 9-year-old female with a height of (149) cm and body weight of (35). On her preoperative examination we have not mentioned any medical abnormalities. General anesthesia was induced, with propofol and rocuronium using a curarisation monitoring. Endotracheal tube was introduced after a TOF of 0 at the level of the eyebrow. The ESP block was performed as follows: The patient was placed in lateral left position, while respecting the rules of asepsis. A high-frequency linear ultrasound transducer (9-12 MHz linear probe (LOGIQE; GE Healthcare, Wauwatosa, WI, USA) was placed in a longitudinal parasagittal orientation, $2 \mathrm{~cm}$ lateral to the T6

Corresponding Author:-Yasser Asmai.

Address:-Pediatric ICU, Children Hospital, Mohamed V University, Faculty of Medicine and Pharmacy, Rabat, Morocco. 
spinous process (fig.1). Three muscles were identified superficial to the hyperechoic transverse process shadow as follows: trapezius, rhomboid major (RMM), and erector spinae muscle (ESM) (fig. 2; A). An 18 Gauge 55 mm insulated needle (Sonoplex stim, Pajunk, Geisingen, Germany) was advanced in cranio-caudal direction further to contact the T6 spinous process in plane of the ultrasound transducer. The deep plane to the erector spinae muscle was identified (fig. 2; B). After negative aspiration, the ESP block was performed by injection of $17,5 \mathrm{ml}$ of $0,25 \%$ bupivacaine into the interfascial plane between erector spinae muscle and T6 spinous process confirmed by visualization of local anesthetic spreading (LA) in a linear pattern and between erector spinae and the bony acoustic shadow of transverse spinous process (fig. 2; C). Considering the fear of the postoperative pain, we have inserted a 20 Gauge $400 \mathrm{~mm}$ continuous peripheral catheter $2 \mathrm{~cm}$ beyond the tip of the needle into the interfascial plane superficial to erector spinae muscle (fig. 3).

Anesthesia was maintained with sevoflurane (MAC 3\%). The perioperative period was uneventful. The patient was stable, and the changes in heart rate and blood pressure did not exceed $10 \%$ of baseline with no opioids needed.

The patient's visual analog scale for pain was $1 / 10$ in the postoperative recovery room immediately after extubation, and no other rescue analgesic treatments required. After 30 min follow-up, the patient was transferred to ward. The visual analog scale of pain controlled hourly, was inferior to $3 / 10$ for the first 24 hours.

\section{Discussion:-}

Analgesia using thoracic epidural catheterization is still considered the gold standard for postoperative pain management in major open abdominal surgeries ${ }^{1}$. However, there are concerns regarding side effects such as hypotension, motor blockade, as well as the risk of major complications such as epidural hematoma and abscess 2 . Recently, Fascial plane blocks, such as transversus abdominis plane (TAP) block, have increased in popularity because of their ease of placement and lower risk of complication than neuroaxial or paravertebral techniques 6 . Nevertheless, they have their own limitations: dermatomal coverage is limited ${ }^{7,8}$ and a recent meta-analysis found that the TAP block to be marginally effective in this context ${ }^{9}$.

ESP block is a novel regional anesthetic technique originally described for thoracic neuropathic pain when performed at the T5 transverse process ${ }^{10}$. Local anesthetic is injected between the erector spinae muscle and transverse process under ultrasound guidance, blocking the dorsal and ventral rami of the thoracic and abdominal spinal nerves ${ }^{11,12}$. Recently, It has been shown to be effective in providing somatic and abdominal analgesia when performed at the T7-9 level ${ }^{13}$. The erector spinae plane block may produce extensive multidermatomal sensory block. In a cadaveric model, an injection of $20 \mathrm{ml}$ of fluid at T7 produced spread extending up to the C7-T2 spinous process cranially, and down to the L2-L3 spinous process caudally ${ }^{14}$.

To demonstrate this potential, we aimed to share our experience of ESP block in a pediatric hepatic hydatid cyst surgery. Considering the subcostal incision in our case, we have suggested to perform our catheterized ESP block at the T6 transverse process. Since the block was accomplished after anesthesia, we were not able to clarify the dermatomal spread of the ESP block. however, no additional bolus of opioids was required as the variability of the blood pressure and heart rate was not important. The perioperative period stability without additional opioids may prove that the required dermatomal coverage for this surgery was achieved.

In TAP Block, Catheter techniques are complicated to perform because of the proximity to the surgical field and the presence of air between the planes that prevent the insertion of a catheter. The ESPB allows postoperative continuous analgesia via catheterization because this anesthetic procedure is performed far of the surgical zone. Also, the catheter can be inserted postoperatively, despite the presence of surgical bandage and disruption of tissue planes by surgery. In our reported case, the placement of catheter was easy. it was connected to an automatic analgesia pump (NIPRO super fuser $250 \mathrm{ml}$ with variable flow regulator) with $0,125 \%$ bupivacaine at $8 \mathrm{ml} / \mathrm{hour}$ and was used to extend the duration of analgesia as needed. The postoperative course was uneventful and our patient had no additional analgesics during the first 24 hours. The catheter was removed on the second postoperative day.

\section{Conclusion:-}

The analgesic benefits of regional anesthesia for pediatric surgery must be carefully weighed against the potential risks, particularly as these blocks are generally performed in the anesthetized patient. ESP block is an attractive alternative because of the ease of realization as the sonographic target is easily visualized, and the safety profile with 
minimal risk. The analgesic efficacy observed in our patient indicates that this technique could be a good alternative to thoracic epidural analgesia in hepatic surgery.

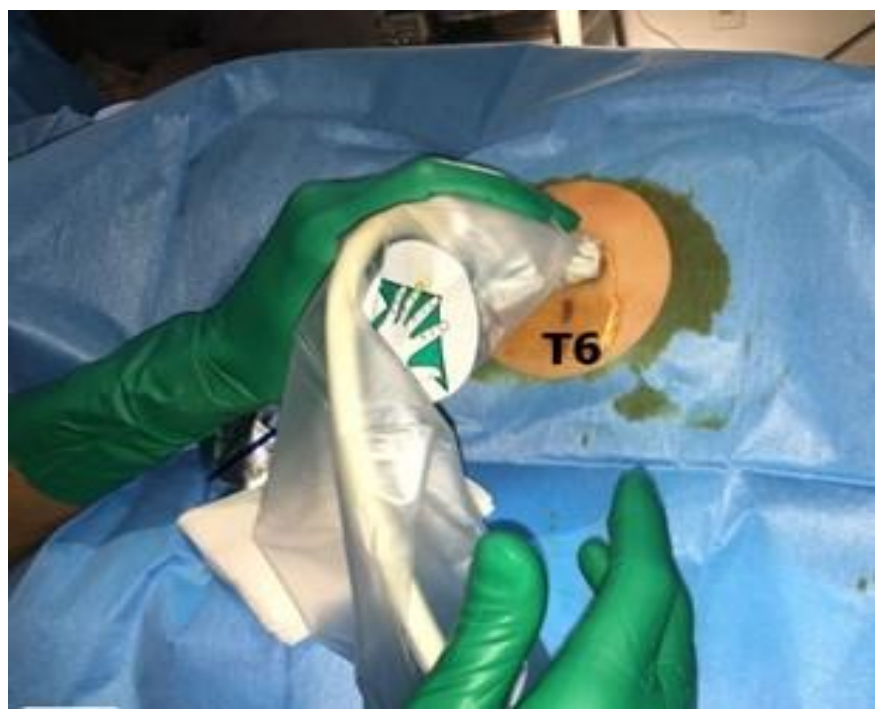

Figure 1:-Probe position for the ESP block with the patient in lateral left decubitus.

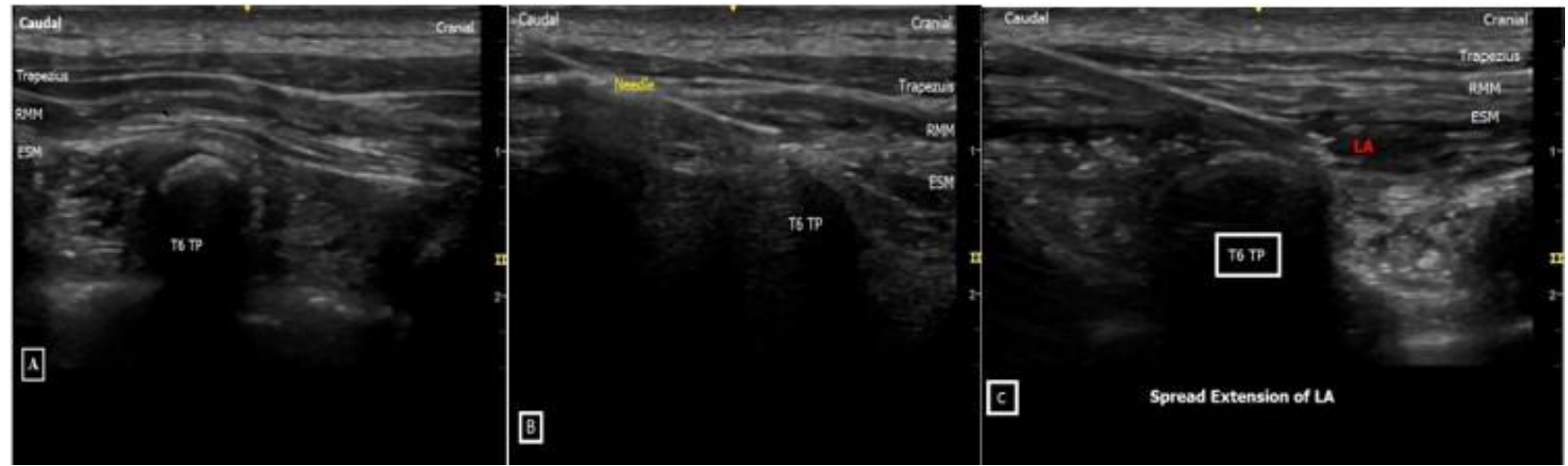

\section{Figure 2:-}

1. Identification of erector spinae muscle superficial to the hyperechoic transverse process shadow.

2. Ultrasound showing needle on top of transverse process of T6.

3. visualization of local anesthetic spreading in a linear pattern between erector spinae muscle and the transverse process of $\mathrm{T} 6$.

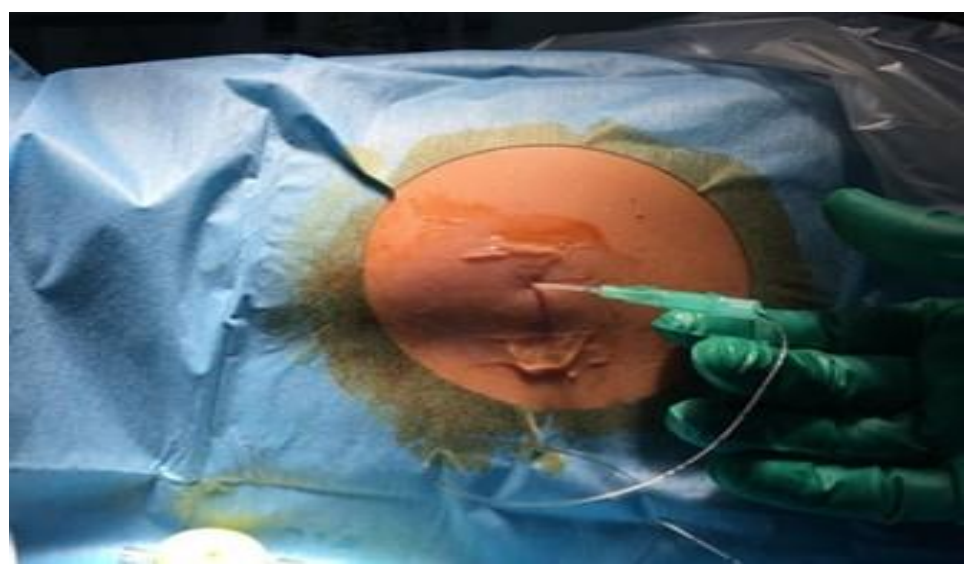

Figure 3:-insertion of a continuous peripheral catheter into the interfascial plane superficial to erector spinae muscle. 


\section{References:-}

1. Guay, Nishimori M, Kopp S. Epidural local anesthetics versus opioid-based analgesic regimens for postoperative gastrointestinal paralysis, vomiting and pain after abdominal surgery. Cochrane Database Syst Rev. 2016; 7: CD 001893

2. Chilvers CR, Nauyen NH, Robertson IK. Changing from epidural to multimodal analgesia for colorectal laparotomy: an audit. Anesth Intensive Care. 2007; 35: 230-238

3. Rawal N. Epidural technique for postoperative pain: gold standard no more? Reg Anesth Pain Med. 2012; 37: 310-317

4. Boezaart AP, Raw RM. Spleeding beauty or big bad wolf? Reg anesth Pain Med. 2006; 31: 189-191

5. Costach I, de Neumann L, Ramnanan CJ, et al. The mid-point. Transverse process to pleura (MTP) block: a new end-point for thoracic paravertebral block. Anaethesia. 2017; 72: 1230-1236

6. EL-Baghdadly K, Pawa A. The Erector Spinae Plane Block: plane and simple. Anaesthesia. 2017; 72: 434-438

7. Chin KJ, McDonnell JG, Carvalho B, Sharkery A, Pawa A, Gadsden J. Essentials of our current understanding: abdominal wall blocks. Reg Anesth Pain Med. 2017; 42: 133-183

8. Niraj G, Kelkar A, Hart E, Kaushik V, Fleet D, Jameson J. Four quadrant transversus abdominis plane block and continuous transversus abdominis plane analgesia: a-\# year prospective audit in 124 patients. J Clin Anesth. 2015; 27: 579-584

9. Baeriswyl M, Kirkham KR, Kern C, Albrecht E. The analgesic efficacy of ultrasound-guided transversus abdominis plane block in adult patients: a meta-analysis. Anesth Analg. 2015; 121:1640-1654.

10. Forero M, Adhikary SD. Lopez H. Tsui C. Chin LJ. The Erector Spinae Plane Block a novel analgesic technique neuropathic pain. Reg Anesth Pain Med 2016: 41; 621-7

11. Ueshima $\mathrm{H}$, Otake $\mathrm{H}$, Clinical experiences of ultrasound-guided erector spinae plane block for thoracic vertebra surgery. J Clin. Anesth. 2017: 38: 137.

12. Ueshima $\mathrm{H}$, Otake $\mathrm{H}$. Erector spinae plane block provides effective pain management during pneumothorax surgery. J Clin Anesth. 2017: 40: 74

13. Chin KJ, Malhas L, Perlas A. The erector spinae plane block provides visceral abdominal analgesia in bariatric surgery: a report of 3 cases. Reg Anesth Pain Med. 2017; 42: 372-376

14. Chin KJ, Adhikary S, Sarwani N, Forero M. The analgesic efficacy of pre-operative bilateral erector spinae plane (ESP) blocks in patients having ventral hernia repair. Anaesthesia. 2017; 72:452-460. 